\title{
Profile and Outcome of Patients with Acute Cholangitis in a Tertiary Center in South India
}

\author{
Prasanth Raghhupatruni ${ }^{1} \quad$ Rajesh Gopalakrishna ${ }^{1, \odot} \quad$ Vinodkumar Ankarath ${ }^{1} \quad$ Shine Sadasivan ${ }^{1}$ \\ ${ }^{1}$ Department of Gastroenterology, Amrita Institute of Medical \\ Sciences, Amrita Viswa Vidyapeetham, Kochi, Kerala, India

\begin{abstract}
Address for correspondence Rajesh Gopalakrishna, MD, DNB, MNAMS, FICP, FRCP, Department of Gastroenterology, Amrita Institute of Medical Sciences, AIMS Ponekkkara PO, Kochi, Kerala, 682041, India (e-mail: grajesh22@gmail.com).
\end{abstract}

\begin{abstract}
Keywords

- ERCP

Background The mainstay of management of acute cholangitis includes endoscopic or percutaneous biliary drainage and antimicrobial therapy. We aimed to study the profile and outcomes among patients with acute cholangitis who underwent endoscopic biliary drainage at our center.

Methods Seventy consecutive patients with acute cholangitis diagnosed and managed as per the Tokyo Guidelines 2018 for acute cholangitis between June 1, 2018 to December 31, 2019 were prospectively studied. Clinical, etiological and microbial profile, therapy, and patient outcomes were analyzed.

Results Choledocholithiasis (54.3\%) and benign biliary stricture (28.6\%) and malignancy $(17.1 \%)$ were common etiological factors. Thirteen patients $(18.6 \%$ ) had underlying chronic liver disease. Moderate-to-severe cholangitis was seen in $67.7 \%$ of patients with high and very high grade as compared with $54.5 \%$ with medium grade of Charlson comorbidity index $(\mathrm{CCl})$ and $41.2 \%$ of patients with low grade of $\mathrm{CCl}$. Elevated C-reactive protein, low albumin, and prolonged international normalized ratio were associated with severe cholangitis. Bile culture was positive in $62.9 \%$ and blood culture was positive in $15.7 \%$ of patients. Bile cultures were predominantly polymicrobial in contrast to blood cultures (53.8 vs. $18 \%$ ). Escherichia coli was the predominant isolate in blood and bile. Multidrug resistant (MDR) organisms were seen in $79.5 \%$ of positive bile cultures.

- PTBD

- bacterobilia

- bile culture

Conclusions A positive blood or bile culture, but not presence of multiple organisms or presence of MDR organisms in bile, was associated with severity of cholangitis. There was no mortality among these patients in-hospital or at 28-days.
\end{abstract}

\section{Introduction}

Acute cholangitis is an infectious disease of the biliary tract with a wide spectrum of presentation ranging in severity from a mild form, with fever and jaundice, to a severe form with septic shock. ${ }^{1}$ It is a potentially life-threatening systemic condition characterized by an infection of the bile, which is normally sterile, and biliary obstruction. It occurs when biliary stenosis, due to various benign causes (often bile duct stones) or the presence of a tumor, resulting in cholestasis
DOI https://doi.org/ $10.1055 / \mathrm{s}-0041-1739561$ ISSN 0976-5042
(C) 2021. Society of Gastrointestinal Endoscopy of India. This is an open access article published by Thieme under the terms of the Creative Commons Attribution-NonDerivative-NonCommercial-License, permitting copying and reproduction so long as the original work is given appropriate credit. Contents may not be used for commercial purposes, or adapted, remixed, transformed or built upon. (https://creativecommons.org/licenses/by-nc-nd/4.0/). Thieme Medical and Scientific Publishers Pvt. Ltd. A-12, 2nd Floor, Sector 2, Noida-201301 UP, India 
and biliary infection. ${ }^{2}$ This condition was first described in 1877 by Charcot as having a triad of right upper abdominal pain, fever, and jaundice. The mainstay of the management of acute cholangitis includes endoscopic or percutaneous biliary drainage and antimicrobial therapy. ${ }^{3}$ Clinically updated and comprehensive epidemiological data on patients with acute cholangitis are limited. Most of the studies were retrospective studies. In this background, the present study is conducted prospectively to diagnose patients with acute cholangitis, assess the severity as per the Tokyo Guidelines 2018 (TG18) for acute cholangitis, and study clinical, microbiological profile and outcomes among the patients presenting to a tertiary care center in south India.

\section{Methods}

Patients aged 18 years or older diagnosed with acute cholangitis and who went underwent endoscopic retrograde cholangiopancreatography (ERCP) between June 1, 2018 and December 31,2019 at our center were prospectively enrolled. Patients who had prior percutaneous biliary access and/or drainage were excluded. The patient demographics, all relevant clinical variables including biochemical, hematological, microbiological investigations, and other relevant data, were recorded on a predesigned proforma. Patients were classified as per the TG18 severity grading as grade I (mild), grade II (moderate), and grade III (severe). Patients were treated as per the TG18 management for acute cholangitis and the hospital stay, intensive care unit (ICU) stay, in-hospital and 28-day mortality were analyzed. Bile cultures were obtained using bile specimens obtained at biliary cannulation at ERCP after discarding initial $3 \mathrm{~mL}$ bile. Blood cultures were also obtained in most patients.

Statistical analysis was performed using IBM SPSS version 20.0 software. Categorical variables were expressed using frequency and percentage. Numerical variables were presented using mean and standard deviation. Chi-squared test with continuity correction was used to test the statistical significance of the association of all parameters between severity grading. Independent sample $t$-test was used to study the statistical significance of the comparison of age and severity grading. A $p$-value of $<0.05$ was considered to be statistically significant.

\section{Results}

Seventy (53 males, 17 females) consecutive patients admitted with acute cholangitis between June 1, 2018 and December 31, 2019 were included in the study. Patient characteristics are shown in - Table 1. The mean age of the patients was $51.57 \pm 16.2$ years. Only 20 (28.6\%) of the patients presented with classical Charcot's triad of pain, fever, and jaundice. In patients who presented with classical Charcot's triad, 14 (70\%) patients had moderate-to-severe cholangitis, while $6(30 \%)$ patients had mild cholangitis.

TG18 for acute cholangitis were used for diagnosis and management. Out of 70 patients, 30 patients (42.8\%) were classified as grade I (mild), 36 patients $(51.4 \%)$ as grade
II (moderate), and 4 patients (5.7\%) as grade III (severe). Moderate-to-severe cholangitis was significantly associated with elevated C-reactive protein (CRP) $(p$-value $<0.001)$, total bilirubin ( $p$-value $<0.001$ ), and alkaline phosphatase (ALP) ( $p$-value $=0.029)$; and also with deranged international normalized ratio (INR) $(p$-value $=0.039)$ and hypoalbuminemia $(p$-value $<0.001)$.

Thirteen patients (18.6\%) had underlying chronic liver disease (CLD). About $76.9 \%$ of patients with CLD had moderate-to-severe cholangitis, whereas $52.6 \%$ of patients without CLD had moderate-to-severe cholangitis (see - Fig. 1). Thirty-one (44.3\%) patients were in high and very high grades of CCI. About $67.7 \%$ of patients with high and very high grade of $\mathrm{CCI}$ had moderate-to-severe cholangitis, whereas $54.5 \%$ of patients with medium grade of $\mathrm{CCI}$ had moderate-to-severe cholangitis and $41.2 \%$ of patients with low grade of CCI had moderate-to-severe cholangitis (see - Fig. 2).

Fifty-nine patients (84.3\%) had no growth on blood culture and 11 patients (15.7\%) had growth on blood culture. Most common organisms grown on blood culture are Escherichia. coli (63.6\%) Klebsiella (27.3\%), and Enterococcus species (9.1\%). In patients who had growth on blood cultures $90.9 \%$ had moderate-to-severe cholangitis. Among patients with moderate-to-severe cholangitis, $50.8 \%$ had no growth on blood cultures (see - Fig. 3A).

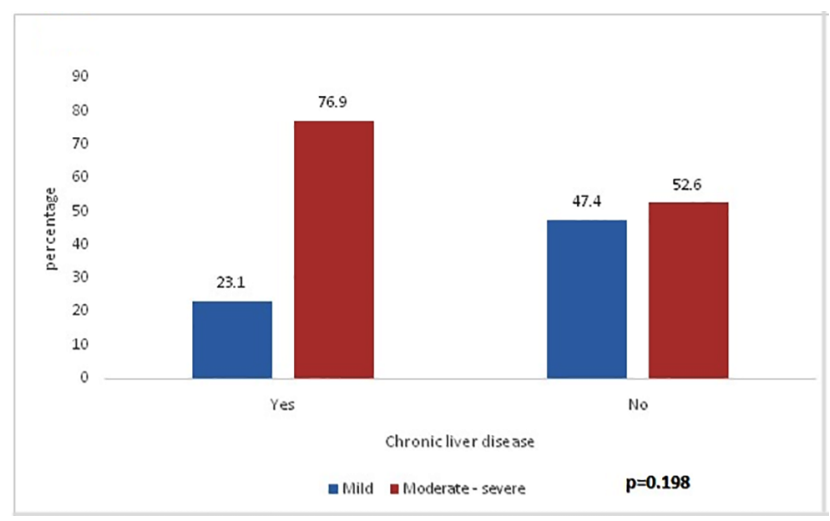

Fig. 1 Association of chronic liver disease with severity of cholangitis.

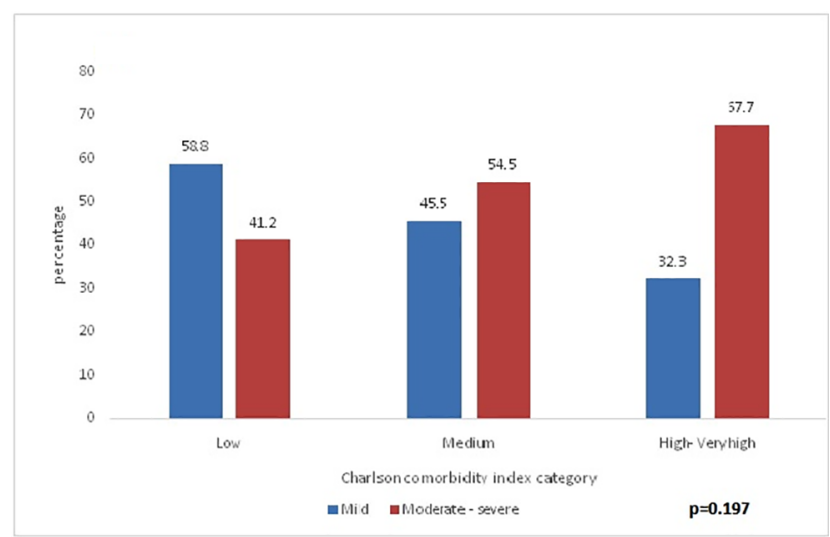

Fig. 2 Association of Charlson comorbidity index with severity of cholangitis. 
Table 1 Characteristics of patients with acute cholangitis who underwent ERCP and managed as per TG18

\begin{tabular}{|c|c|c|c|}
\hline & Mild $(n=30)$ & Moderate-to-severe $(n=40)$ & $p$-Value \\
\hline Mean age $(y)$ & 49.8 & 52.9 & 0.43 \\
\hline Males: females & $22: 8$ & $31: 9$ & 0.68 \\
\hline \multicolumn{4}{|l|}{ Etiology } \\
\hline Benign biliary stricture $(n=20)$ & 9 & 11 & \\
\hline Choledocholitihiasis $(n=38)$ & 18 & 20 & \\
\hline Malignant stricture $(n=12)$ & 3 & 9 & \\
\hline Patients with CLD $(n=13)$ & 3 & 10 & 0.19 \\
\hline Patients with DM $(n=20)$ & 10 & 10 & 0.44 \\
\hline \multicolumn{4}{|l|}{$\mathrm{CCl}$} \\
\hline $\operatorname{Low}(n=17)$ & 10 & 7 & \\
\hline Medium $(n=22)$ & 10 & 12 & \\
\hline High $(n=19)$ & 5 & 14 & \\
\hline Very high $(n=12)$ & 5 & 7 & \\
\hline \multicolumn{4}{|l|}{ Laboratory parameters: median (IQR) } \\
\hline Hemoglobin (g/dL) & $11.50(1.9)$ & $10.75(2.68)$ & 0.22 \\
\hline WBC count $\left(10^{9} / \mathrm{L}\right)$ & $9.25(4.21)$ & $8.98(7.18)$ & 0.51 \\
\hline Platelets (109/L) & $250.5(139.5)$ & $228.0(158.0)$ & 0.146 \\
\hline CRP (mg/L) & $16(39.3)$ & $66.4(90.0)$ & $<0.001$ \\
\hline Bilirubin (mg/dL) & $1.59(2.56)$ & $3.52(7.12)$ & 0.001 \\
\hline Albumin (gm/dL) & $3.75(0.93)$ & $3.15(1.05)$ & $<0.001$ \\
\hline SGOT (units/L) & $31.7(62.1)$ & $41.4(43.4)$ & 0.12 \\
\hline SGPT (units/L) & $4.1(88.38)$ & $40.0(87.9)$ & 0.79 \\
\hline $\operatorname{ALP}(I U / L)$ & $156.5(165)$ & $275.0(311.5)$ & 0.02 \\
\hline INR & $0.91(0.17)$ & $1.04(0.29)$ & 0.03 \\
\hline Creatinine (mg/dL) & $0.78(0.33)$ & $0.86(0.40)$ & 0.18 \\
\hline
\end{tabular}

Abbreviations: ALP, alkaline phosphatase; CCI, Charlson comorbidity index; CLD, chronic liver disease; CRP, C-reactive protein; DM, diabetes mellitus; ERCP, endoscopic retrograde cholangiopancreatography; INR, international normalized ratio; IQR, interquartile range; SCOT, serum glutamic oxaloacetic transaminase; SGPT, serum glutamic pyruvic transaminase; TG18, Tokyo Guidelines 2018; WBC, white blood count.

Out of 70 patients, 39 (55.7) patients had growth on bile culture, while 23 patients had no growth on bile culture and 8 patients had missing bile cultures. In patients who had growth on bile cultures, $71.8 \%$ had moderate-to-severe cholangitis. Among the patients who had no growth on bile cultures, only $24.8 \%$ had moderate-to-severe cholangitis (see - Fig. 3B). Out of 39 patients who had bile culture growth, $21(53.8 \%)$ patients had multiple organisms. The most common organisms grown on bile culture were E. coli (46.2\%), Enterococcus (46.2\%), and Klebsiella species (41\%). In patients with $E$. coli growth in the bile, $72.2 \%$ had moderate-to-severe cholangitis, whereas in patients with Klebsiella species 81.8\% had moderate-to-severe cholangitis and in patients with Enterococcus species $83.3 \%$ had moderate-to-severe cholangitis. In patients who had growth of multiple organisms on bile cultures, $76.2 \%$ had moderate-to-severe cholangitis, whereas $66.7 \%$ had moderate-to-severe cholangitis in patients who had single organism growth on bile cultures (see - Fig. 4A). Out of 39 patients who had bile culture growth, 31 (79.5\%) patients had multidrug resistance (MDR) organisms. In patients who had growth of MDR organisms on bile cultures, $74.2 \%$ had moderate-to-severe cholangitis, whereas $62.5 \%$ had moderate-to-severe cholangitis in patients who had non-MDR organism growth on bile cultures (see - Fig. 4B).

Nine out of twelve (75\%) patients with malignant biliary stricture had moderate-to-severe cholangitis, while 11/20 (55\%) patients with benign biliary stricture and 20/38 (52.6\%) with choledocholithiasis had moderate-to-severe cholangitis. Out of 70 patients, biliary stenting was done in 66 patients. All the four patients, in whom biliary stenting was not done at ERCP, had mild cholangitis. Out of 70 patients, four patients had failed endoscopic drainage, requiring repeat intervention (percutaneous transhepatic biliary drainage [PTBD] in 3 and ERCP in 1) within 28 days. All four patients with failed endoscopic drainage, requiring repeat intervention had moderate-to-severe cholangitis

There was no mortality while admitted in the hospital or on follow-up for 28 days. Eighteen out of thirty (60\%) patients with mild cholangitis had hospital stay $>5$ days, while $33 / 40$ 


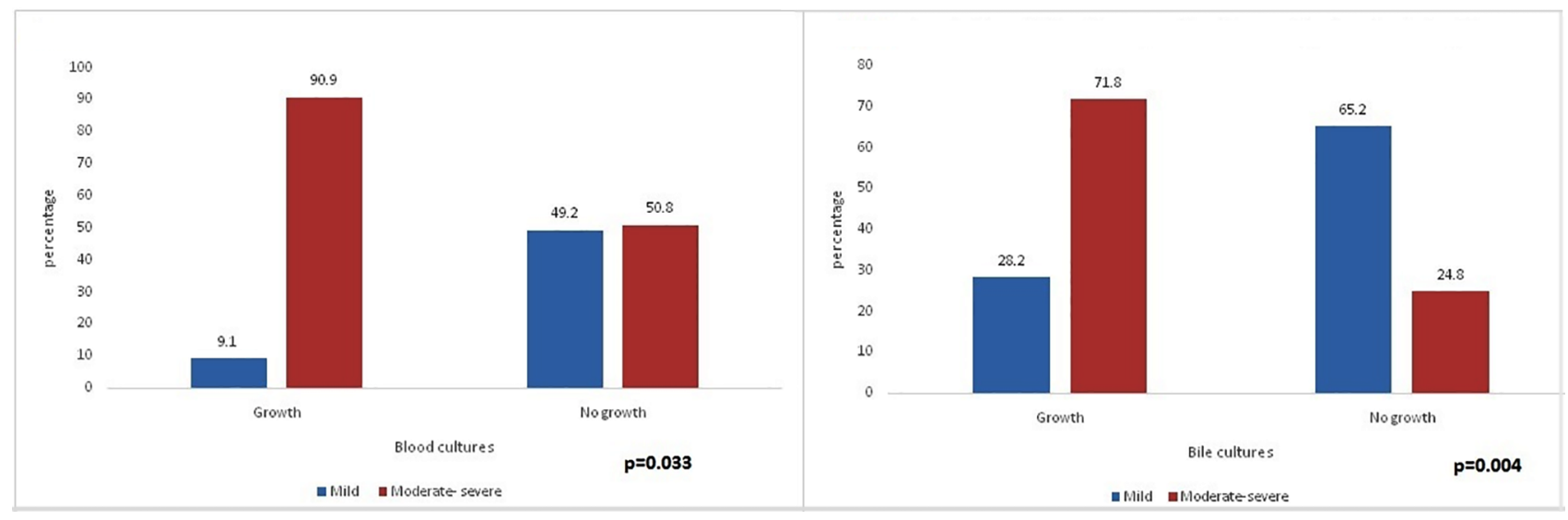

Fig. 3 (A) Association of blood culture growth with severity of acute cholangitis. (B) Association of bile culture growth with severity of acute cholangitis.

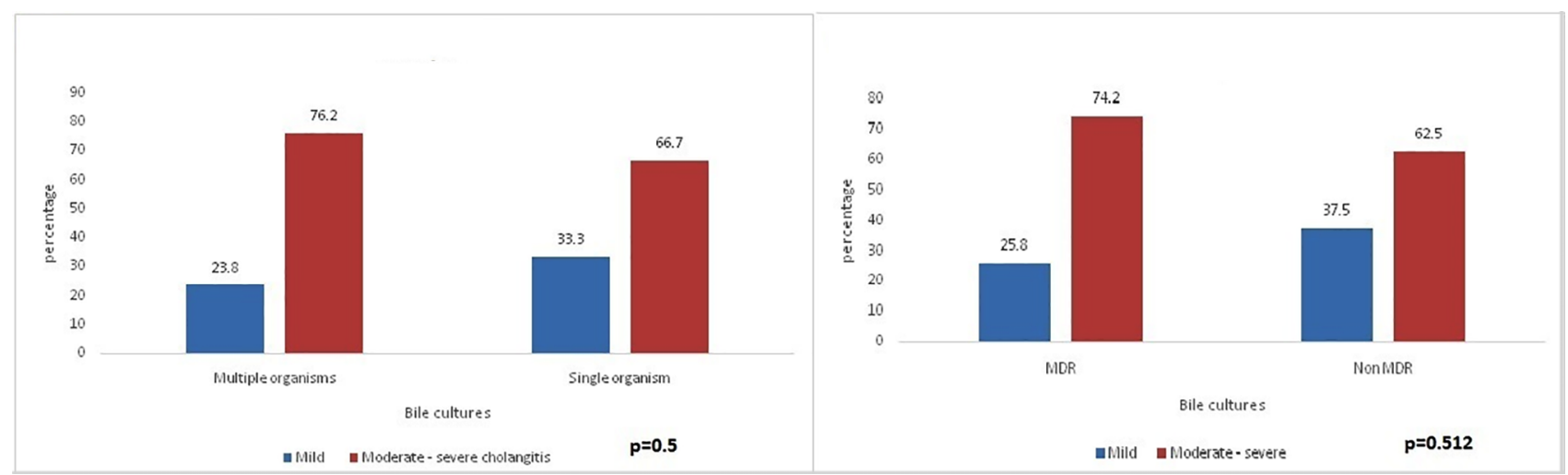

Fig. 4 (A) Association of growth of multiple organisms in bile culture with severity of acute cholangitis. (B) Association of growth of multidrug resistant (MDR) organisms in bile culture with severity of acute cholangitis.

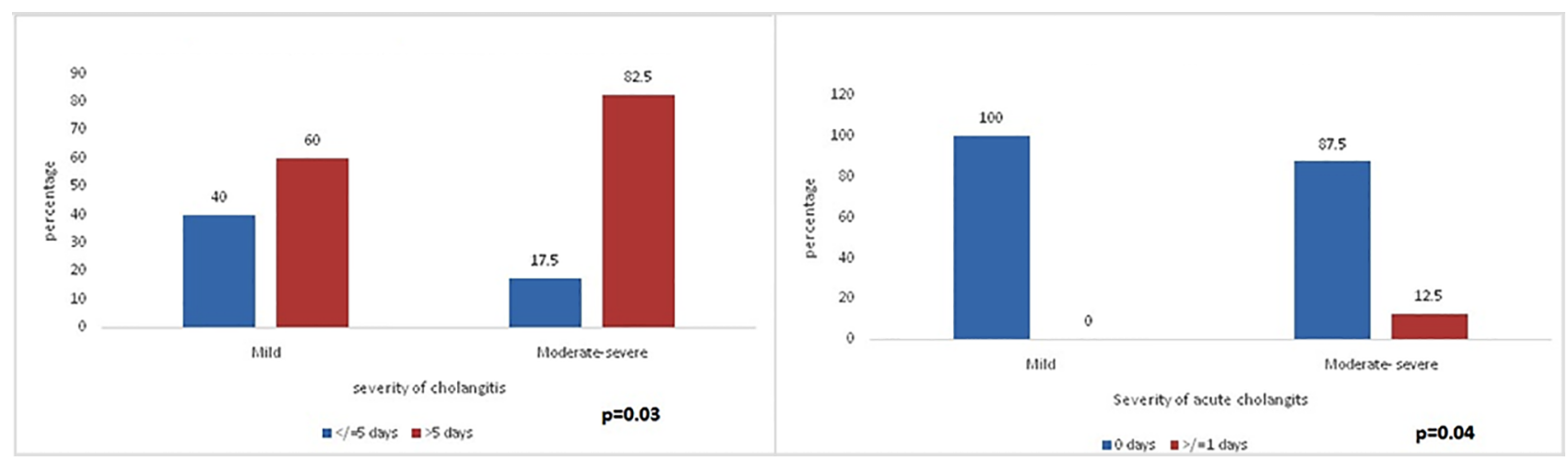

Fig. 5 (A) Association of severity of cholangitis with length of total hospital stay. (B) Association of severity of cholangitis with length of intensive care unit stay.

(82.5\%) of patients with moderate-to-severe cholangitis had hospital stay $>5$ days (see $\mathbf{- F i g}$. $\mathbf{5 A}$ ). None of the patients with mild cholangitis needed ICU care, while $5(12.5 \%)$ of patients with moderate-to-severe cholangitis needed ICU stay (see - Fig. 5B). Mean ICU stay was 2.4 days.

\section{Discussion}

In our study, 30 patients (42.8\%) presented with Tokyo 2018 severity grade I, 36 (51.4\%) patients with grade II, and
4 patients (5.7\%) with grade III cholangitis. In comparison to the multicenter retrospective observational study by Gomi et $\mathrm{al}^{3}{ }^{3}$ of 6,433 patients, where $37.5,36.2$, and $26.2 \%$ were in TG13 grades I, II, and III, respectively, our study had higher proportions of patients in grade I and II, but less of grade III. Hence, we have classified grades II and III together as moderate-to-severe cholangitis and grade I as mild cholangitis, for further statistical analysis.

The mean age of the patients in our study is 51.5 years that is similar to an Indian study by Sahu et al, ${ }^{4}$ where mean age 
was 51.3 years, and another study by Shenoy et al, ${ }^{5}$ where the mean age was 47.6 years. In our study, the mean age of mild cholangitis group was 49.8 years, whereas it was 52.9 years in moderate-to-severe group. Two-third of the patients aged more than 60 years presented with moderate-to-severe cholangitis and the rest with mild cholangitis. There was a trend of elderly patients presenting with more severe forms of cholangitis. The large multicenter study by Gomi et $\mathrm{al}^{3}$ in Japan and Taiwan also has shown significant association of advanced age with severe forms of cholangitis.

Choledocholithiasis was the most common cause (54.3\%) of biliary obstruction and cholangitis in our study. Benign biliary stricture is the second most common cause seen in $28.6 \%$ of the patients followed by malignant biliary stricture in $17.1 \%$ of the patients. In the study by Gomi et $\mathrm{al}^{3}$ in Japan and Taiwan, the majority had bile duct stones, followed by either biliary stent obstruction or biliary tumors. Boey and Way $^{6}$ in a series from the West also reported stone disease as the predominant cause of biliary obstruction. Retrospective study done by Sahu et al in India reported choledocholithiasis as the most common cause (62\%) followed by malignant obstruction (30\%) and benign bile duct stricture (9\%). ${ }^{4}$ Our study has shown more patients (75\%) with malignant biliary stricture presenting with moderate-to-severe cholangitis compared with stone disease (52.6\%) and benign biliary stricture (55\%). But the association was not statistically not significant.

Several studies also had analysis on the relation of Charcot's triad to the severity of acute cholangitis with varying results. Kiriyama et $\mathrm{al}^{7}$ found no association between Charcot's triad and the severity of acute cholangitis, and Gigot et al found no association between Charcot's triad and mortality outcomes. In contrast, Tsuyuguchi et al reported an increased sensitivity of Charcot's triad with disease severity, ${ }^{8}$ and O'Connor et $\mathrm{al}^{9}$ found that the prevalence of Charcot's triad was higher in participants who survived. Our study has shown that $70 \%$ of the patients who had classical Charcot's triad at presentation had moderate-to-severe cholangitis, whereas in patients who did not present with Charcot's triad 52\% had moderate-to-severe cholangitis. But the association was not significant ( $p$-value 0.169).

Laboratory tests in acute cholangitis typically reveal leucocytosis with shift to left and cholestatic pattern of liver function. In our patients, mean white blood cell (WBC) count is 9,630 cells $/ \mathrm{mL}$ in patients with mild cholangitis, whereas it is 11,060 cells $/ \mathrm{mL}$ in patients with moderate-to-severe cholangitis. Mean hemoglobin is $11.3 \mathrm{~g} / \mathrm{dL}$ in patients with mild cholangitis, whereas it is $10.7 \mathrm{~g} / \mathrm{dL}$ in patients with moderate-to-severe cholangitis. Both suggest a trend of leucocytosis and anemia associated with more severe forms of cholangitis. But the association was not statistically significant. In the study done by Sahu et $\mathrm{al}^{4}{ }^{4}$ mean hemoglobin was similar $(11.1 \mathrm{~g} / \mathrm{dL})$ to our study, but the mean WBC count was higher (13,800 cells/mL).

Many studies have shown that thrombocytopenia is associated with acute severe cholangitis. Wada et $\mathrm{a}^{10}$ have proposed platelet count less than 1 lakh/ $\mu \mathrm{L}$, as one of the criteria for diagnosing severe cholangitis, in the Tokyo guidelines for diagnosis and severity grading of acute cholangitis in 2007 . This was approved even in the revised Tokyo guidelines in 2013 and 2018. But our study has shown no significant association of severity of cholangitis with thrombocytopenia with mean platelet counts of 2.61 and 2.26 lakhs $/ \mu \mathrm{L}$ in mild and moderate-to-severe cholangitis, respectively.

Our study has shown that hyperbilirubinemia is associated with severity of cholangitis. Mean value of total bilirubin was $2.51 \mathrm{mg} / \mathrm{dL}$ in mild cholangitis group, whereas it was $6.3 \mathrm{mg} / \mathrm{dL}$ in moderate-to-severe cholangitis group ( $p$-value 0.001). Retrospective study done by Schwed et al had also shown similar association. ${ }^{11}$ Patients with adverse events had mean bilirubin of $7.1 \mathrm{mg} / \mathrm{dL}$, whereas those without adverse events had a mean bilirubin of $4.4 \mathrm{mg} / \mathrm{dL}$. It was demonstrated in that study that total bilirubin level $>10 \mathrm{mg} / \mathrm{dL}$ in patients with acute cholangitis is predictor of death or organ failure. Mean ALP in our study was $200.67 \mathrm{IU} / \mathrm{L}$ in mild cholangitis group and $305.75 \mathrm{IU} / \mathrm{L}$ in moderate-to-severe cholangitis group suggesting that a higher degree of cholestasis is associated with an increased likelihood of poor outcome ( $p$-value 0.029). In the retrospective study done by Sahu et $\mathrm{al}^{4}{ }^{4}$ mean ALP was higher than $371.50 \mathrm{IU} / \mathrm{L}$. In our study, there was no significant association of transaminases with severity of cholangitis.

Hypoalbuminemia had significant association with severity of cholangitis ( $p$-value $<0.001$ ) in our study group. Mean serum albumin value in patients with mild cholangitis is $3.71 \mathrm{~g} / \mathrm{dL}$, whereas in patients with moderate-to-severe cholangitis it is $3.08 \mathrm{~g} / \mathrm{dL}$. Retrospective analysis by Tsuyuguchi et $\mathrm{al}^{8}$ also has shown similar results with mean albumin values of $3.8,3.5$, and $2.9 \mathrm{~g} / \mathrm{dL}$ in mild, moderate, and severe groups, respectively. It was also suggested by the group that in cases of acute cholangitis with hypoalbuminemia but without organ dysfunction, early endoscopic drainage should be performed without waiting for the outcome of conservative medical treatment. The results of this study showed that hypoalbuminemia $(<2.8 \mathrm{~g} / \mathrm{dL})$ and increased prothrombin time-international normalized ratio (PT-INR) $(>1.5)$ were statistically significant predictors of mortality. According to the TG07, increased PT-INR occurs because of organ dysfunction. In such cases, urgent biliary drainage was recommended. In our study, mean INR value in patients with mild cholangitis is 1.00 , whereas in patients with moderate-to-severe cholangitis it is 1.12 suggesting that coagulopathy is associated with severe forms of cholangitis ( $p$-value 0.039 ).

CRP is a biomarker and is frequently used for clinical testing to indicate the presence of severe sepsis. High concentrations of serum CRP are closely related with the high risk of organ failure and death. CRP value may be a useful factor in severity assessment of cholangitis as shown in that of acute pancreatitis; ${ }^{11}$ however, in the study by Tsuyuguchi et al cutoff value (CRP $>5.0 \mathrm{mg} / \mathrm{dL}$ ) based on the receiver operating characteristic analysis did not show ideal sensitivity or specificity. ${ }^{7}$ Whereas our study has shown that high CRP levels in the blood, it is associated with severe forms of cholangitis. Mean CRP value in patients with mild cholangitis is $30.3 \mathrm{mg} / \mathrm{L}$, whereas in patients with moderate-to-severe cholangitis, it is $85.5 \mathrm{mg} / \mathrm{L}(p$-value $<0.001)$. 
In most of the studies, the predominant organisms isolated from both blood and bile were gram negative with $E$. coli being the most frequent isolate.in bile cultures. ${ }^{12,13}$ In our study, E. coli (46.2\%) and Enterococcus spp (46.2\%) were the most common organisms, followed by Klebsiella spp (41\%). E. coli and Klebsiella spp were the most common organisms grown on bile cultures in another retrospective Indian study by Shenoy et al. ${ }^{5}$

Our study has shown that patients with severe forms of cholangitis have longer ICU stay and prolonged hospital stay. In our study, most of the patients (92.8\%) did not require ICU care, whereas only five patients $(7.2 \%)$ required ICU stay for 1 or more than 1 day. None of the patients with mild cholangitis had ICU, whereas $12.5 \%$ of patients with moderate-to-severe cholangitis had ICU stay for 1 or more than 1 day. About $72.8 \%$ of the patients had hospital stay more than 5 days. The proportion is high (82.5\%) in moderate-to-severe cholangitis group compared with mild cholangitis (60\%). All our patients were followed up for 28 days and there were no in-hospital or 28-day mortality.

A limitation of our study was that the frequency of anaerobes, such as Bacteroides and Clostridia, and fungi, is probably underestimated because we used standard culture techniques in our study. However, our study showed a significant proportion of Enterococcus species in bile cultures as compared with previous reports.

In conclusion, we found a higher proportion of moderate-to-severe cholangitis in patients with malignant strictures as compared with benign causes. However, this may be related to increased age of patients with malignant strictures. A positive blood culture was associated with moderate-to-severe cholangitis. A positive bile culture growth, but not polymicrobial growth or presence of MDR organisms, was associated with moderate-to-severe cholangitis. There was no mortality among the patients in-hospital or at 28-days in this study.

\section{Ethics Statement}

Written informed consent was obtained from all the study subjects. The study protocol conforms to the ethical guidelines of the "World Medical Association (WMA) Declaration of Helsinki Ethical Principles for Medical Research Involving Human Subjects" adopted by the 18th
WMA General Assembly, Helsinki, Finland, June 1964, as revised in Tokyo 2004. The study was approved by the institutional ethical review committee.

\section{Conflict of Interest}

All authors declare that they have no conflict of interest.

\section{References}

1 Lipsett PA, Pitt HA. Acute cholangitis. Surg Clin North Am 1990;70(6):1297-1312

2 Kiriyama S, Kozaka K, Takada T, et al. Tokyo Guidelines 2018: diagnostic criteria and severity grading of acute cholangitis (with videos). J Hepatobiliary Pancreat Sci 2018;25(1):17-30

3 Gomi H, Takada T, Hwang TL, et al. Updated comprehensive epidemiology, microbiology, and outcomes among patients with acute cholangitis. J Hepatobiliary Pancreat Sci 2017;24(6):310-318

4 Sahu MK, Chacko A, Dutta AK, Prakash JA. Microbial profile and antibiotic sensitivity pattern in acute bacterial cholangitis. Indian J Gastroenterol 2011;30(5):204-208

5 Shenoy SM, Shenoy S, Gopal S, Tantry BV, Baliga S, Jain A. Clinicomicrobiological analysis of patients with cholangitis. Indian J Med Microbiol 2014;32(2):157-160

6 Boey JH, Way LW. Acute cholangitis. Ann Surg 1980;191(3):264-270

7 Kiriyama S, Takada T, Hwang TL, et al. Clinical application and verification of the TG13 diagnostic and severity grading criteria for acute cholangitis: an international multicenter observational study. J Hepatobiliary Pancreat Sci 2017;24(6):329-337

8 Tsuyuguchi T, Sugiyama H, Sakai Y, et al. Prognostic factors of acute cholangitis in cases managed using the Tokyo Guidelines. J Hepatobiliary Pancreat Sci 2012;19(5):557-565

9 O'Connor MJ, Schwartz ML, McQuarrie DG, Sumer HW. Acute bacterial cholangitis: an analysis of clinical manifestation. Arch Surg 1982;117(4):437-441

10 Wada K, Takada T, Kawarada Y, et al. Diagnostic criteria and severity assessment of acute cholangitis: Tokyo Guidelines. J Hepatobiliary Pancreat Surg 2007;14(1):52-58

11 Schwed AC, Boggs MM, Pham XD, et al. Association of admission laboratory values and the timing of endoscopic retrograde cholangiopancreatography with clinical outcomes in acute cholangitis. JAMA Surg 2016;151(11):1039-1045

12 Yeom DH, Oh HJ, Son YW, Kim TH. What are the risk factors for acute suppurative cholangitis caused by common bile duct stones? Gut Liver 2010;4(3):363-367

13 Motte S, Deviere J, Dumonceau JM, Serruys E, Thys JP, Cremer M. Risk factors for septicemia following endoscopic biliary stenting. Gastroenterology 1991;101(5):1374-1381 